\title{
Home Health Aid
}

National Cancer Institute

\section{Source}

National Cancer Institute. Home Health Aid. NCI Thesaurus. Code C53429.

A person trained to for provide personal patient care and related services for ill or disabled patients, and elderly in home or home-like settings to get patients needs met. The tasks performed by home health aide include personal care, homemaking, communications, working with the patient occupational therapist, physical therapist and other caregivers to assure that the patients are reaching their best physical, mental and social well-being. 